\title{
Lung adenocarcinoma expression profile: one more layer of heterogeneity
}

\author{
Elisabeth Brambilla
}

Affiliation: INSERM U823, Institute Albert Bonniot, University Joseph Fourier, and Dept of Pathology, Grenoble University Hospital, Grenoble, France.

Correspondence: Elisabeth Brambilla, Dept of Pathology, Grenoble University Hospital, CS 10217, 38043 Grenoble Cedex 09, France. E-mail: EBrambilladchu-grenoble.fr

0 @ERSpublications

New study of gene expression profiles sheds light on the diversity and heterogeneity of lung adenocarcinoma http://ow.ly/oQys8

Adenocarcinoma exhibits the highest level of heterogeneity among human tumours, with at least five patterns being admixed in the majority of them. The 2004 World Health Organization classification recognised this heterogeneity under the term of mixed type adenocarcinoma, which accounted for $85 \%$ of histopathological diagnoses by pathologists. Thus, this classification was not rewarding at the level of histogenesis, biological underlying properties and prognostic prediction. To shed light onto this obscure heterogeneity, the International Multidisciplinary Lung Adenocarcinoma Classification (International Association for the Study of Lung Cancer/European Respiratory Society/American Thoracic Society) was established by an international multidisciplinary panel of pathologists, clinicians, oncologists, pulmonologists, molecular biologists, radiologists and surgeons, with the ambitious task of discriminate five subtypes based on architectural patterns and their predominance (percentage of each in each case, a 5\% increment) [1]. Histological subtyping in lepidic, acinar, papillary solid and micropapillary patterns was proposed in order to increase the visibility of adenocarcinoma in the molecular arena and to establish correlation between adenocarcinoma predominant subtypes and clinico-pathological and prognostic characteristics. Therefore, this classification has important implications in the staging of tumours, introducing adenocarcinoma in situ and minimally invasive adenocarcinoma, both with $100 \%$ 5-year survival expectancy after resection. Adenocarcinoma in situ presents with pure ground-glass opacity (GGO) radiologically and an absence of histological invasion. Minimally invasive adenocarcinoma is characterised by an invasion area less than $5 \mathrm{~mm}$ and radiological (computed tomography scan) density on the background of GGO. This classification was also needed to provide a uniform terminology based on objective diagnostic criteria, and thus suppress the terms bronchioloalveolar carcinoma and mixed subtype adenocarcinoma, which both lack reproducibility, clinical counterparts and prognostic value.

Histo-radiological correlations and surgical implications for patients management have been emphasised [1-4]. Strategy for optimal approaches to diagnosis of advanced lung cancer patients on small biopsies or cytology fitting new treatment requirements was proposed. The prognostic value of this adenocarcinoma classification was internationally confirmed [4-11].

In the current issue of the European Respiratory Journal, FukUr et al. [12] report on the identification of the basal cell $(\mathrm{BC})$ gene signature in lung adenocarcinoma and its prognostic value. Their data provide evidence that the $\mathrm{BC}$ transcriptome they previously established [13] contributes unique molecular features of airway $\mathrm{BCs}$ to the heterogeneity of adenocarcinoma. This subtype of lung adenocarcinoma, which expresses high levels of airway BC genes, is associated with aggressive clinical outcome. This BC transcriptome should correlate with the centrally located adenocarcinoma thought to arise from the surface or glandular epithelium of bronchi, in contrast to the terminal respiratory unit (TRU) adenocarcinoma previously

Received: May 222013 | Accepted after revision: Sept 122013

Conflict of interest: None declared.

Copyright @ERS 2013 
described, for which the stem cells are likely to be exocrine bronchiolar cells (Clara cells) and type II pneumocytes $[14,15]$. The BC signature should overlap that of non-terminal respiratory unit (NTRU) signature of TAKEUCHI et al. [14]; however, the authors did not propose any comparison for similitude level between their BC signature and the NTRU signature, despite the fact that they have quite common gene composition. Indeed over-representation of cell cycle related pathways and Ras mutation are common characteristic features of NTRU and the adenocarcinoma with high BC signature. In contrast, TRU profile displays high expression of NKX2-1 gene encoding for TTF1 transcription factor, and a predominance of EGF-R mutations.

Using the airway BC signature (862 genes) previously characterised in the author's laboratory [13] a BC index $\left(\mathrm{I}_{\mathrm{BC}}\right)$ was calculated for each individual subject, allowing categorisation of subjects as BC-low or BChigh individuals. The primary cohort (182 patients) [16] was followed by two validation cohorts. The relationship with survival was exceptional between BC-low signature adenocarcinoma and those with BChigh signature (high expression: fall change $\geqslant 5, \mathrm{p}=0.01$ ) by genome-wide microarray comparison. Furthermore, Kaplan-Meier analysis of the primary training set and of the two validation test sets allowed recognition of significantly shorter survival in those patients with $B C$-high $(p=0.007$ and $p=0.01)$ when compared to BC-low signature with the log-rank test. In multivariate analysis using Cox regression analysis introducing the main variable of cell survival in lung adenocarcinoma, airway BC signature was as powerful as pathological stage and age to predict shorter survival (HR 1.59, 95\% CI 1.14-2.22). In univariate analysis, BC-high signature significantly correlated with low frequency of lepidic pattern $(\mathrm{p}<0.001)$ high pathological grade $(p<0.001)$ and vascular invasion $(p<0.004)$. Importantly, the NKX2 gene (encoding TTF1) was significantly downregulated in BC-high versus BC-low adenocarcinoma $(\mathrm{p}<0.001)$. This study was well designed since there are at least three candidate stem cells for the development of lung cancer, and bronchial BCs are believed to provide a progenitor for squamous cell carcinoma and small cell lung carcinoma. Although the candidate stem cells for adenocarcinoma in the peripheral airways have been proposed based on the mouse model to be exocrine bronchiolar cells [17] or type II cells expressing TTF1, the progenitor stem cell for proximal non-terminal unit adenocarcinoma remains elusive. The debate is still open as to whether the histogenetic origin of the central adenocarcinoma arises from another stem cell progenitor.

Previous to this analysis several signatures have been found to be prognostically pertinent. That of SHEDDEN et al. [18] was re-examined at the level of gene enrichment for functional pathways by BRYANT et al. [19]. In this Director Challenge analysis, as well as further validations on the Japanese and European database of Affymetrix expression analysis, it was suggested that the functional pathways enriched in adenocarcinoma depended on the predominant pattern of the new adenocarcinoma classification [5]. Adenocarcinoma with cell cycle upregulation and downregulation of immune genes demonstrated significantly poorer outcome, as did those with a predominant solid component per se. Additionally, the presence of a solid component (whatever size) predicted decreased survival as compared to tumours without a solid component. Consistent with the $\mathrm{BC}$ signature correlation with the absence of lepidic pattern, the lepidic predominant pattern had better prognosis.

From a practical point of view, the new adenocarcinoma classification offers several aspects of prognostic value: lepidic pattern predominant adenocarcinoma has the best prognosis, with $86 \% 5$-year survival, whereas solid and micropapillary pattern have dismal prognosis, with less than 50\% 5-year survival. Only two predominant patterns are still intermediate and not well defined as regard to prognosis (acinar and papillary pattern) [4-11].

The present article raises several questions about the BC signature. Are the BC-high adenocarcinoma with higher frequency of KRAS mutation, activation of cell cycle pathway, a suppression or downregulation of signature of exocrine bronchiolar cell genes (NKX2) comparable to solid type adenocarcinoma which mostly share these features? Are these BC-high adenocarcinomas TTF1 negative, which should accelerate and facilitate their recognition? In other words, is there a way to select these patients on histo-phenotypical characteristics that could confer a clinical utility to the BC signature? As with adenocarcinomas with EML4/ ALK fusions, which share TTF1 and P63 phenotype, are mucin secreting and probably derive from a junctional cell between bronchioli and alveolar epithelium, do the BC-high adenocarcinomas more often express P63? The epithelial-mesenchymal transition (EMT) activation pathway described by the authors fosters testing of the phenotypical traits of EMT properties on the series of BC-high transcriptome, such as Snail or Slug/ZEB1 expression and the switch from E-cadherin to N-cadherin suggested by the authors.

Finally, considering that the patterns described histologically in the new classification [5] were highly predictive of survival after stratification per stage in the different series in the USA, Australia, Japan and Europe, is BC signature in adenocarcinoma competing with the predictive prognostic power of solid and micropapillary patterns? Had the variables solid and micropapillary patterns been introduced in the multivariate analysis, we would know! Unfortunately with the exception of 104 subjects analysed in 
Memorial Sloan Kettering Cancer Center, most of the cases were studied in silico and not reviewed in a centralised way by an expert pathologist.

Although the main actors of the BC high signature are not considered yet as oncogenic drivers, targeting them could really help to understand their impact in tumour development, a need quite unmet in most adenocarcinoma, since driver's targetable mutations have been detected in only half of them. As elegantly proposed by the authors, KRAS will be soon targetable (still a target untreatable with drugs), and MYC and cyclin-dependent kinases (cell cycle deregulation), as well as extracellular matrix genes, may represent additional approaches to induce synthetic lethality interactions in BC-high adenocarcinoma. Strikingly, the relationship between tumour cells and cellular matrix constitution is gaining interest with the discovery of the DDR2 (discovering domain receptor 2) mutation, a receptor to collagen 4, in the upper and lower airway, especially in smokers [20].

We would have expected a BC signature corresponding to a typical basal airway cell to be more efficient in distinguishing subtypes of squamous cell carcinoma, which is not the case since the authors themselves recognise that the signature is shared by all squamous cell carcinoma because of their common derivation from airway basal epithelial cells.

Altogether, this paper sheds new light on the diversity and heterogeneity of lung adenocarcinoma, and raises interest in and fosters the integration of expression signatures into common morpho-phenotypic analysis in clinical use for prognostic and predictive value of customised therapy efficacy.

\section{References}

1 Lederlin M, Puderbach M, Muley T, et al. Correlation of radio- and histomorphological pattern of pulmonary adenocarcinoma. Eur Respir J 2013; 41: 943-951.

2 Van Schil PE, Asamura H, Rusch VW, et al. Surgical implications of the new IASLC/ATS/ERS adenocarcinoma classification. Eur Respir J 2012; 39: 478-486.

Austin JH, Garg K, Aberle D, et al. Radiologic implications of the 2011 classification of adenocarcinoma of the lung. Radiology 2013; 266: 62-71.

4 Maeyashiki T, Suzuki K, Hattori A, et al. The size of consolidation on thin-section computed tomography is a better predictor of survival than the maximum tumour dimension in resectable lung cancer. Eur J Cardiothorac Surg 2013; 43: 915-918.

5 Travis WD, Brambilla E, Noguchi M, et al. International Association for the Study of Lung Cancer/American Thoracic Society/European Respiratory Society international multidisciplinary classification of lung adenocarcinoma. J Thorac Oncol 2011; 6: 244-285.

6 Travis WD, Brambilla E, Van Schil P, et al. Paradigm shifts in lung cancer as defined in the new IASLC/ATS/ERS lung adenocarcinoma classification. Eur Respir J 2011; 38: 239-243.

7 Yoshizawa A, Motoi N, Riely GJ, et al. Impact of proposed IASLC/ATS/ERS classification of lung adenocarcinoma: prognostic subgroups and implications for further revision of staging based on analysis of 514 stage I cases. Mod Pathol 2011; 24: 653-664.

8 Russel PA, Wainer Z, Wright GM, et al. Does lung adenocarcinoma subtype predict patient survival? A clinicopathologic study based on the new International Association for the Study of Lung Cancer/American Thoracic Society/European Respiratory Society international multidisciplinary lung adenocarcinoma classification. J Thorac Oncol 2011; 6: 1496-1504.

9 Yoshizawa A, Sumiyoshi S, Sonobe M, et al. Validation of the IASLC/ATS/ERS lung adenocarcinoma classification for prognosis and association with EGFR and KRAS gene mutations: analysis of 440 Japanese patients. $J$ Thorac Oncol 2013; 8: 52-61.

10 Warth A, Muley T, Meister M, et al. The novel histologic International Association for the Study of Lung Cancer/ American Thoracic Society/European Respiratory Society classification system of lung adenocarcinoma is a stageindependent predictor of survival. J Clin Oncol 2012; 30: 1438-1446.

$11 \mathrm{Xu} \mathrm{L}$, Tavora F, Battafarano R, et al. Adenocarcinomas with prominent lepidic spread: retrospective review applying new classification of the American Thoracic Society. Am J Surg Pathol 2012; 36: 273-282.

12 Fukui T, Shaykhiev R, Agosto-Perez F, et al. Lung adenocarcinoma subtypes based on expression of human airway basal cell genes. Eur Respir J 2013; 42: 1332-1344.

13 Hackett NR, Shaykhiev R, Walters MS, et al. The human airway epithelial basal cell transcriptome. PLoS ONE 2011; 6: e18378.

14 Takeuchi T, Tomida S, Yatabe Y, et al. Expression profile-defined classification of lung adenocarcinoma shows close relationship with underlying major genetic changes and clinicopathologic behaviors. J Clin Oncol 2006; 24: 1679-1688. Yatabe Y. EGFR mutations and the terminal respiratory unit. Cancer Metastasis Rev 2010; 29: 23-36.

16 Chitale D, Gong Y, Taylor BS, et al. An integrated genomic analysis of lung cancer reveals loss of DUSP4 in EGFRmutant tumors. Oncogene 2009; 28: 2773-2783.

17 Kim CF, Jackson EL, Woolfenden AE, et al. Identification of bronchioalveolar stem cells in normal lung and lung cancer. Cell 2005; 121: 823-835.

18 Shedden K, Taylor JM, Enkemann SA, et al. Gene expression-based survival prediction in lung adenocarcinoma: a multi-site, blinded validation study. Nat Med 2008; 14: 822-827.

19 Bryant CM, Albertus DL, Kim S, et al. Clinically relevant characterization of lung adenocarcinoma subtypes based on cellular pathways: an international validation study. PLoS ONE 2010; 5: e11712.

20 Hammerman PS, Sos ML, Ramos AH, et al. Mutations in the DDR2 kinase gene identify a novel therapeutic target in squamous cell lung cancer. Cancer Discov 2011; 1: 78-89. 